Bull. Austral. Math. Soc.

Vol. 40 (1989) [49-57]

\title{
ON WITT'S DIMENSION FORMULA FOR FREE LIE ALGEBRAS AND A THEOREM OF KLYACHKO
}

\author{
D. Blessenohl and H. LaUe
}

\begin{abstract}
It is shown that Witt's basic dimension formula and a more recent result of Klyachko imply each other. Then Klyachko's identities between certain idempotents in the group ring of $S_{n}$ are supplemented by identities involving Wever's classical idempotent. This leads to a direct proof of Klyachko's theorem (and hence Witt's formula), avoiding any commutator collecting process. Furthermore, this approach explains why the Witt dimensions are numbers which otherwise occur when "counting necklaces".
\end{abstract}

\section{INTRODUCTION}

A classical result in Lie theory is Witt's formula for the dimensions of the homogeneous subspaces of a finitely-generated free Lie algebra $L$ : If $H$ is the subspace of $L$ generated by all homogeneous elements of multidegree $\left(k_{1}, \ldots, k_{\ell}\right)$, then

$$
\operatorname{dim} H=\frac{1}{n} \sum_{d \mid k_{1}, \ldots, k_{\ell}} \mu(d) \frac{(n / d) !}{\left(k_{1} / d\right) ! \ldots\left(k_{\ell} / d\right) !}
$$

where $n=k_{1}+\ldots+k_{\ell}$.

The subspace of all homogeneous elements of multidegree $(1, \ldots, 1)$ is a module for the symmetric group $S$ on the set of free generators. A result of Klyachko [5] states that, under mild conditions on the scalar ring, this module is isomorphic to $M^{S}$ where $M$ is a faithful irreducible module for the subgroup generated by the longest cycle in $S$.

We establish a short chain of equivalences between these two theorems. The link is given by an application of a theorem from character theory of symmetric groups. At a certain point the well-known "counting of necklaces" comes in. Thus the Witt dimension numbers are embedded in their natural combinatorial surroundings. In the final section we slightly modify and reorganise the ideas of the second part of [5]. In particular, we show that Klyachko's result as described above may be obtained independently of any commutator collecting process. By virtue of our results in the second section, this also holds (at least over $Q$ ) for Witt's dimension formula. Throughout, we work in the lie algebra generated by the free generators of a free associative algebra, but we do not make use of the theorem proved by Witt that this Lie algebra is free.

Received 13 September, 1988

Copyright Clearance Centre, Inc. Serial-fee code: 0004-9729/89 \$A2.00+0.00. 


\section{LINEARISATION OF $A^{Z}\left(k_{1}, \ldots, k_{\ell}\right)$}

Let $R$ be a commutative ring with 1 . For any set $Z$, the free $R$-algebra which is freely generated by $Z$ is denoted by $A^{Z}$. Let $\ell \in \mathrm{N}$, and let $Z$ be a set of $\ell$ elements $z_{1}, \ldots, z_{\ell}$. For all $m \in N$ let $A_{m}^{Z}$ be the $R$-span in $A^{Z}$ of all monomials $z_{i_{1}} \ldots z_{i_{m}}$ of degree $m$. If $k_{1}, \ldots, k_{\ell} \in \mathrm{N}_{0}$, we write $A^{Z}\left(k_{1}, \ldots, k_{\ell}\right)$ for the $R$-span of all monomials whose $z_{j}$-degree is $k_{j}$ for $1 \leqq j \leqq \ell$. In this section we connect these spaces with the group ring of $S_{n}$ over $R$ where $n=k_{1}+\ldots+k_{\ell}$.

Let $X$ be a set of $n$ elements $x_{1}, \ldots, x_{n}$ and $\Sigma$ be the set of all mappings $\varphi: X \rightarrow$ $Z$ with the property $\left|z_{j} \varphi^{-1}\right|=k_{j}$ for $1 \leqq j \leqq \ell . S_{n}$ acts on $X$ by $x_{j} \pi=x_{j \pi}$ for all $j \in\{1, \ldots, n\}, \pi \in S_{n}$. Obviously, $\pi \varphi \in \Sigma$ for all $\pi \in S_{n}$ and $\varphi \in \Sigma$. This yields a transitive action of $S_{n}$ on $\Sigma$. For all $\varphi, \psi \in \Sigma$ we set

$$
Y_{\varphi, \psi}=\left\{\pi \mid \pi \in S_{n}, \quad \pi \varphi=\psi\right\}
$$

Then $Y_{\varphi, \varphi}$ is a Young subgroup of $S_{n}$, corresponding to the decomposition $\left(k_{1}, \ldots, k_{\ell}\right)$ of $n$; hence $Y_{\varphi, \varphi} \cong S_{k_{1}} \times \ldots \times S_{k_{\ell}}$. As $Y_{\varphi, \varphi}$ is the stabiliser of $\varphi$ in $S_{n}$, we have $|\Sigma|=n ! /\left(k_{1} ! \ldots k_{\ell} !\right)$. If $\pi \in Y_{\varphi, \psi}$, then $Y_{\varphi, \psi}=\pi \cdot Y_{\varphi, \varphi}$. We set

$$
\mathcal{A}=A^{X}(1, \ldots, 1)
$$

and for fixed $\varphi \in \Sigma$ we define the $R$-linear mapping

$$
\Lambda_{\varphi}: A^{Z}\left(k_{1}, \ldots, k_{\ell}\right) \rightarrow \mathcal{A}
$$

by $\left(\left(x_{1} \psi\right) \ldots\left(x_{n} \psi\right)\right) \Lambda_{\varphi}=\sum_{\pi \in \breve{Y}_{\varphi, \psi}} x_{1 \pi} \ldots x_{n \pi}$ for all $\psi \in \Sigma$. The function $\Lambda_{\varphi}$ may be viewed as a "complete linearisation" of the elements of $A^{Z}\left(k_{1}, \ldots, k_{\ell}\right)$. As the sets $Y_{\varphi, \psi}(\psi \in \Sigma)$ are left cosets of $Y_{\varphi, \varphi}$ and $S_{n}$ is their disjoint union, the elements $\sum_{\pi \in Y_{\varphi, \psi}} x_{1 \pi} \ldots x_{n \pi}(\psi \in \Sigma)$ are $R$-linear independant. Hence the linearisation $\Lambda_{\varphi}$ is injective.

The $R$-space $A^{Z}\left(k_{1}, \ldots, k_{\ell}\right)$ is an $R S_{n}$-left module via the $R$-linear extension of the $S_{n}$-action

$$
\pi\left(z_{i_{1}} \ldots z_{i_{n}}\right)=\tilde{z}_{i_{1 \pi}} \ldots \tilde{z}_{i_{n \pi}}
$$

(see $[2,(2),(3)])$. In the same manner, $\mathcal{A}$ also is an $R S_{n}$-left module. We prove

$$
\Lambda_{\varphi} \text { is an } R S_{n}-\text { left module homomorphism: }
$$


Proof: For all $r, \sigma \in S_{n}, \psi \in \Sigma$, we have (by $[2,(8)]$ )

$$
\begin{aligned}
\tau\left(\left(\left(x_{1} \psi\right) \ldots\left(x_{n} \psi\right)\right) \Lambda_{\varphi}\right) & =\sum_{\sigma \in Y_{\varphi, \psi}} x_{1 \tau \sigma} \ldots x_{n \tau \sigma}=\sum_{\sigma \in \tau Y_{\varphi, \psi}} x_{1 \sigma} \ldots x_{n \sigma} \\
=\sum_{\sigma \in Y_{\varphi, \tau \psi}} x_{1 \sigma} \ldots x_{n \sigma} & =\left(\left(x_{1} \tau \psi\right) \ldots\left(x_{n} \tau \psi\right)\right) \Lambda_{\varphi}=\left(\left(x_{1 \tau} \psi\right) \ldots\left(x_{n \tau} \psi\right)\right) \Lambda_{\varphi} \\
& =\left(\tau\left(\left(x_{1} \psi\right) \ldots\left(x_{n} \psi\right)\right)\right) \Lambda_{\varphi}
\end{aligned}
$$

hence (1).

$\mathcal{A}$ is a (regular) $R S_{n}$-right module via the $R$-linear extension of the $S_{n}$-action

$$
\left(x_{1 \sigma} \ldots x_{n \sigma}\right) \tau=x_{1 \sigma \tau} \ldots x_{n \sigma \tau} \quad\left(\sigma, \tau \in S_{n}\right)
$$

$(\operatorname{see}[2,(9)])$.

$$
\text { If } k_{1} ! \ldots k_{\ell} \text { ! is a unit in } R, \text { then im } \Lambda_{\varphi} \text { is the space of fixed elements }
$$
under the right action of $Y_{\varphi, \varphi}$ on $\mathcal{A}$.

Before we prove (2), we introduce the notation $\bar{W}=\sum_{\sigma \in W} \sigma \in R S_{n}$ for all $W \subseteq S_{n}$ and remark

$$
\begin{gathered}
\left(\left(x_{1} \psi\right) \ldots\left(x_{n} \psi\right)\right) \Lambda_{\varphi}=\left(x_{1 \pi} \ldots x_{n \pi}\right) \bar{Y}_{\varphi, \varphi} \text { for all } \psi \in \Sigma, \pi \in Y_{\varphi, \psi}, \\
\text { as }\left(\left(x_{1} \psi\right) \ldots\left(x_{n} \psi\right)\right) \Lambda_{\varphi}=\left(x_{1} \ldots x_{n}\right) \bar{Y}_{\varphi, \psi}=\left(x_{1} \ldots x_{n}\right) \overline{\pi Y}_{\varphi, \varphi}=\left(x_{1 \pi} \ldots x_{n \pi}\right) \bar{Y}_{\varphi, \varphi} .
\end{gathered}
$$
Hence im $\Lambda_{\varphi}=\mathcal{A} \bar{Y}_{\varphi, \varphi}$ and im $\Lambda_{\varphi}$ consists of fixed elements under the right action of $Y_{\varphi, \varphi}$. If $a \in \mathcal{A}$ is a fixed point under the right action of $Y_{\varphi, \varphi}$, then

$$
a=\left(\left(k_{1} ! \ldots k_{\ell} !\right)^{-1} a\right) \bar{Y}_{\varphi, \varphi} \in \operatorname{im} \Lambda_{\varphi} .
$$

This proves (2).

We now turn to the Lie multiplication $\circ$ in $A^{Z}$ ( $A^{X}$ respectively), defined by $a \circ b=a b-b a$. Let $L^{Z}$ ( $L^{X}$ respectively) be the Lie algebra generated by $Z$ ( $X$ respectively). (By Witt's theorem, it is freely generated by $Z$ ( $X$ respectively), but this result will not be needed in this paper.) We set

$$
\begin{gathered}
L^{Z}\left(k_{1}, \ldots, k_{\ell}\right)=L^{Z} \cap A^{Z}\left(k_{1}, \ldots, k_{\ell}\right), \\
\mathcal{L}=L^{X} \cap \mathcal{A}, \quad L_{m}^{X}=L^{X} \cap A_{m}^{X} .
\end{gathered}
$$

Then, by [2, (13)], $\mathcal{L}$ is a submodule of the $R S_{n}$-right module $\mathcal{A}$. As in [2], we use the left normed notation for Lie monomials. We have 
(4)

$$
\left(\left(x_{1} \psi\right) \circ \ldots \circ\left(x_{n} \psi\right)\right) \Lambda_{\varphi}=\left(x_{1 \pi} \circ \ldots \circ x_{n \pi}\right) \bar{Y}_{\varphi, \varphi} \text { for all } \psi \in \Sigma, \pi \in Y_{\varphi, \psi}
$$

Proof: Let

$$
\omega_{n}=(i d-(n, \ldots, 1)) \ldots(i d-(2,1))=\prod_{j=n}^{2}(i d-(j, \ldots, 1))
$$

as in $[6,(4)]$. Then

$$
\begin{aligned}
\left(\left(x_{1} \psi\right) \circ \ldots \circ\left(x_{n} \psi\right)\right) \Lambda_{\varphi} & =\left(\omega_{n}\left(\left(x_{1} \psi\right) \ldots\left(x_{n} \psi\right)\right)\right) \Lambda_{\varphi} \text { by }[2,(11)] \\
& =\omega_{n}\left(\left(\left(x_{1} \psi\right) \ldots\left(x_{n} \psi\right)\right) \Lambda_{\varphi}\right) \text { by }(1) \\
& =\omega_{n}\left(\left(x_{1 \pi} \ldots x_{n \pi}\right) \bar{Y}_{\varphi, \varphi}\right) \text { by }(3) \\
& =\left(\omega_{n}\left(x_{1 \pi} \ldots x_{n \pi}\right)\right) \bar{Y}_{\varphi, \varphi} \text { by }[2,(7)] \\
& =\left(x_{1 \pi} \circ \ldots \circ x_{n \pi}\right) \bar{Y}_{\varphi, \varphi} \text { by }[2,(11)]
\end{aligned}
$$

Hence (3) remains valid if the associative nultiplication is replaced by the Lie product, that is, (4). Therefore, the proof of (2) also works for the associated Lie structures, yielding

If $k_{1} ! \ldots k_{\ell} !$ is a unit in $R$, then $L^{Z}\left(k_{1}, \ldots, k_{\ell}\right) \Lambda_{\varphi}$ is the space of fixed elements under the right action of $Y_{\varphi, \varphi}$ on $\mathcal{L}$. .

As $L^{Z}\left(k_{1}, \ldots, k_{\ell}\right)$ is generated by the elements $\left(x_{1} \psi\right) \circ \ldots \circ\left(x_{n} \psi\right)(\psi \in \Sigma), \mathcal{L}$ by the elements $x_{1 \pi} \circ \ldots \circ x_{n \pi}\left(\pi \in S_{n}\right)$, we have

$$
L^{Z}\left(k_{1}, \ldots, k_{\ell}\right) \Lambda_{\varphi}=\mathcal{L} \bar{Y}_{\varphi, \varphi} .
$$

Hence $\Lambda_{\varphi}$ induces also an injective linearisation of Lie elements (see, for example, $[\mathbf{1}$, $4.2])$.

\section{ConNections BetWeen (WDF) AND (Ind)}

In this section let $R$ be a field of characteristic 0 containing a primitive $n$-th root of unity $\varepsilon$. The character of $S_{n}$ with respect to the right module $\mathcal{L}$ will be denoted by $\chi_{\mathcal{L}}$, and for any group $G$, we write $1_{G}$ for the trivial character of $G$. If $\chi, \chi^{\prime}$ are characters of $G$ over $R$, we write $\left(\chi, \chi^{\prime}\right)_{G}$ for their usual scalar product. 
LEMMA 2.1. Let $Y$ be a Young subgroup of $S_{n}$ of type $S_{k_{1}} \times \ldots \times S_{k_{\ell}}$ where $n=k_{1}+\ldots+k_{\ell}$. Then $\operatorname{dim}_{R} L^{Z}\left(k_{1}, \ldots k_{\ell}\right)=\left(\chi_{\mathcal{L}}, 1_{Y}^{S_{n}}\right)_{S_{n}}$.

Proof: Let $\Sigma$ be as in Section 1 and $\varphi \in \Sigma$. As $\Lambda_{\varphi}$ is injective, we have, by (5), $\operatorname{dim}_{R} L^{Z}\left(k_{1}, \ldots k_{\ell}\right)=\operatorname{dim}_{R} C_{\mathcal{C}}\left(Y_{\varphi, \varphi}\right)$. But

$$
\operatorname{dim}_{R} C_{\mathcal{L}}\left(Y_{\varphi, \varphi}\right)=\left(\left.\chi_{\mathcal{L}}\right|_{Y_{\varphi, \varphi}}, 1_{Y_{\varphi, \varphi}}\right)_{Y_{\varphi, \varphi}}=\left(\chi_{\mathcal{L}}, 1_{Y_{\varphi, \varphi}}^{S_{n}}\right)_{S_{n}}
$$

by the Frobenius reciprocity theorem. Now the lemma follows, as $Y_{\varphi, \varphi}$ and $Y$ are conjugate in $S_{n}$.

LemMA 2.2. Let $T$ be a transitive cyclic subgroup of $S_{n}, \lambda$ a faithful irreducible character of $T$ over $R$, and let $Y$ be as in Lemma 2.1. Let $\#_{T}$ be the number of orbits of length $n$ with respect to the right action of $T$ on the set of right cosets of $Y$ in $S_{n}$. Then

$$
\left(\lambda^{S_{n}}, 1_{Y}^{S_{n}}\right)_{S_{n}}=\#_{T}
$$

Proof: Let $\mathcal{S}$ be a set of representatives of the set of double cosets $Y x T\left(x \in S_{n}\right)$. Using the Frobenius reciprocity law, Maschke's theorem and the orthogonality relations we conclude that

$$
\begin{aligned}
\left(\lambda^{S_{n}}, 1_{Y}^{S_{n}}\right)_{S_{n}} & =\left(\lambda,\left.1_{Y}^{S_{\mathfrak{n}}}\right|_{T}\right)_{T} \\
& =\left(\lambda, \sum_{s \in \mathcal{S}}\left(\left.1_{Y}^{s}\right|_{Y^{s} \cap T}\right)^{T}\right)_{T} \\
& =\sum_{s \in \mathcal{S}}\left(\lambda, 1_{Y^{s} \cap T}^{T}\right)_{T} \\
& =\sum_{s \in S}\left(\lambda \mid Y^{s} \cap T, 1_{Y^{\prime} \cap T}\right)_{Y^{\prime} \cap T} \\
& =\left|\left\{s \mid s \in \mathcal{S}, \quad Y^{s} \cap T=1\right\}\right|
\end{aligned}
$$

where $1_{Y}^{s}$ is the class function on $Y^{*}$ which is "conjugate" to $1_{Y}$ via $s$, that is, $1_{Y}$. The condition $Y^{\bullet} \cap T=1$ means that $|Y s T|=|Y| \cdot|T|$ which holds if and only if $\{T s t \mid t \in T\}$ is an orbit of length $n$ with respect to the right action of $T$ on the set of right cosets of $Y$ in $S_{n}$. Thus the proof is complete.

The following interpretation of the numbers which occur in Witt's dimension formula is well-known $([3,2.1 .21])$.

LEMMA 2.3 .

$$
\#_{T}=\frac{1}{n} \sum_{d \mid k_{1}, \ldots, k_{\ell}} \mu(d) \frac{(n / d) !}{\left(k_{1} / d\right) ! \ldots\left(k_{\ell} / d\right) !} .
$$

Our lemmas show the equivalence of Witt's dimension formula 
(WDF)

$$
\operatorname{dim}_{R} L^{Z}\left(k_{1}, \ldots, k_{\ell}\right)=\frac{1}{n} \sum_{d \mid k_{1}, \ldots, k_{\ell}} \mu(d) \frac{(n / d) !}{\left(k_{1} / d\right) ! \ldots\left(k_{\ell} / d\right) !}
$$

for all $k_{1}, \ldots, k_{\ell} \in N$ such that $k_{1}+\ldots+k_{\ell}=n$.

with the statement that

$$
\left(\chi_{\mathcal{L}}, 1_{Y}^{S_{n}}\right)_{S_{n}}=\left(\lambda^{S_{n}}, 1_{Y}^{S_{n}}\right)_{S_{n}} \text { for all Young subgroups } Y \text { of } S_{n}
$$
where $\lambda$ is the character corresponding to a faithful irreducible $R T$-module $M, T$ as in Lemma 2.2 .

Applying $[4,2.2 .10]$, we can continue our chain of equivalences by

$$
\chi_{\mathcal{L}}=\lambda^{S_{n}}
$$

which means that

$$
\mathcal{L} \cong M^{S_{n}}
$$

This last statement was first proved (in a more general setting), and plays a central role in [5]. On the one hand, our chain of equivalences may be viewed as a new proof of (Ind), based on (WDF). On the other hand, they show that any independent proof of (Ind) conversely leads to (WDF). Klyachko's proof in [5] is, however, based on [5, Proposition 1], involving Hall bases and other non-trivial methods. In our final section we show that a certain analysis and rearrangement of Kylachko's ingenious methods in the second part of [5] suffices to give a simpler proof of (Ind).

\section{IDENTITIES IN $R S_{n}$}

In this section let $R$ be a commutative ring with 1 , let $t$ be trancendental over $R$, and let $R(t)$ be the ring of polynomial fractions over $R$ with denominators whose leading coefficients do not divide 0 . We use the terminology for algebras of Section 1 , indicating by an upper bar when we consider algebras over $R(t)$ instead of $R$.

If $r \in \mathrm{N}$, we have the standard left action of $S_{r}$ on $\underbrace{\bar{A}^{X} \times \ldots \times \bar{A}^{X}}_{r}$ given by

$$
\sigma\left(u_{i_{1}}, \ldots, u_{i_{r}}\right)=\left(u_{i_{1 \sigma}}, \ldots, u_{i_{r \sigma}}\right)
$$


for all $\sigma \in S_{r}$ and $u_{i_{j}} \in \bar{A}^{X}$. If $\left(m_{1}, \ldots, m_{r}\right) \in \mathrm{N}^{r}$ and $u_{j} \in \bar{A}_{m_{j}}^{X} \backslash\{0\}$ for $1 \leqq j \leqq r$, we define

$$
\delta\left(j,\left(u_{1}, \ldots, u_{r}\right)\right)=m_{1}+\ldots+m_{j} .
$$

Following Klyachko [5], we define, for all $\sigma \in S_{r}$, setting $\mathrm{u}=\left(u_{1}, \ldots, u_{r}\right)$,

$$
\operatorname{Ind}(\sigma, \mathrm{u})=\sum_{\substack{1 \leq j<r \\ j \sigma>(j+1) \sigma}} \delta(j, \sigma \mathrm{u})
$$

and

$$
K_{\left(m_{1}, \ldots, m_{r}\right)} \mathrm{u}=\sum_{\sigma \in S_{r}} \frac{t^{\operatorname{Ind}(\sigma, \mathrm{u})}}{\prod_{1 \leqq j<r}\left(1-t^{\delta(j, \sigma \mathrm{u})}\right)} u_{1 \sigma} \ldots u_{r \sigma}
$$

if $u_{j} \neq 0$ for all $j$. Moreover, we set $K_{\left(m_{1}, \ldots, m_{r}\right)} \mathbf{u}=0$ if $u_{j}=0$ for any index $j$. Then $K_{\left(m_{1}, \ldots, m_{r}\right)}$ is multilinear, and as in [5] one has

LemMA 3.1. Let $r \in \mathrm{N},\left(m_{1}, \ldots, m_{r}\right) \in \mathrm{N}^{r}$ and $\left(u_{1}, \ldots, u_{r}\right) \in \bar{A}_{m_{1}}^{X} \times \ldots \times \bar{A}_{m_{r}}^{X}$, $1 \leqq i<r$. Then

$$
\begin{aligned}
K_{\left(m_{1}, \ldots, m_{r}\right)}\left(u_{1}, \ldots, u_{r}\right)-K_{\left(m_{1}, \ldots, m_{i+1}, m_{i}, \ldots, m_{r}\right)} & \left(u_{1}, \ldots, u_{i+1}, u_{i}, \ldots, u_{r}\right) \\
& =K_{\left(m_{1}, \ldots, m_{i}+m_{i+1}, \ldots, m_{r}\right)}\left(u_{1}, \ldots, u_{i} \circ u_{i+1}, \ldots, u_{r}\right) .
\end{aligned}
$$

For all $m \in N$ and $\sigma \in S_{m}$, let ind $\sigma=\sum_{\substack{1 \leqq j<m \\ j \sigma>(j+1) \sigma}} j$. If $i_{1}, \ldots, i_{m-1}$ are the unique nonnegative integers such that $0 \leqq i_{j} \leqq j$ and $\sigma=(2,1)^{i_{1}}(3,2,1)^{i_{2}} \ldots(m, \ldots, 1)^{i_{m-1}}$, then it is easily seen that ind $\sigma=i_{1}+i_{2}+\ldots+i_{m-1}$. We set

$$
\kappa_{m}=\sum_{\sigma \in S_{m}} \frac{t^{\text {ind } \sigma}}{\prod_{1 \leqq j<m}\left(1-t^{j}\right)} \sigma \in R(t) S_{m} .
$$

If $u=\left(x_{i_{1}}, \ldots, x_{i_{r}}\right) \in X^{r}$, then $\delta(j, u)=j$ for $1 \leqq j<r$, and $\operatorname{Ind}(\sigma, u)=$ ind $\sigma$, hence

$$
K_{(1, \ldots, 1)}\left(x_{i_{1}}, \ldots, x_{i_{r}}\right)=\kappa_{r} x_{i_{1}} \ldots x_{i_{r}} .
$$

Starting from this equation, one proves inductively by means of 3.1

Lemma 3.2. (See [5, Proposition 3].) If $\left(m_{1}, \ldots, m_{r}\right) \in \mathbb{N}^{r}, m=m_{1}+\ldots+m_{r}$ and $u_{i} \in \bar{L}_{m_{i}}^{X}$ for $1 \leqq i \leqq r$, then

$$
K_{\left(m_{1}, \ldots, m_{r}\right)}\left(u_{1}, \ldots, u_{r}\right)=\kappa_{m} u_{1} \ldots u_{r}
$$

(where the righthand side is defined via the usual left action of $R(t) S_{m}$ on $\bar{A}_{m}^{X}$, see $(0))$.

In particular, the case $r=1$ yields 
CorollaRY 3.3. If $m \in N$ and $u \in \bar{L}_{m}^{X}$ then $\kappa_{m} u=u$.

By a further specialisation we obtain the following identity in the group ring of $S_{n}$ over $R(t)$

TheOREM 3.4. $\kappa_{n} \omega_{n}=\omega_{n}$ (where $\omega_{n}$ is as in $[6,(4)]$, see after (4)).

PROOF: $\omega_{n} x_{1} \ldots x_{n}=x_{1} \circ \ldots \circ x_{n}$, hence $\kappa_{n} \omega_{n} x_{1} \ldots x_{n}=\omega_{n} x_{1} \ldots x_{n}$, by 3.3 . This implies via $[2,(8)]$ the claim of the theorem.

As is seen from the definition of $\kappa_{n}$, the theorem states the following identity in the group ring of $S_{n}$ over $R[t]$

$$
\sum_{\sigma \in S_{n}} t^{\mathrm{ind} \sigma} \sigma \omega_{n}=\prod_{1 \leqq j<n}\left(1-t^{j}\right) \omega_{n}
$$

Let $\mathcal{X} \subseteq S_{n}$ be the set of all cycle products

$$
\left(j_{1}, \ldots, 1\right)\left(j_{2}, \ldots, 1\right) \ldots\left(j_{i}, \ldots, 1\right)
$$

where $n \geqq j_{1}>j_{2}>\ldots>j_{i} \geqq 2$ and $0 \leqq i<n$. Then $\omega_{n}=\sum_{\pi \in \mathcal{X}}(-1)^{1 \pi^{-1}} \pi$. Comparing coeflicients in (9), we see that (9) is equivalent to

$$
\sum_{\sigma^{-1} \in \mathcal{X}}(-1)^{1 \sigma+1 \pi^{-1}} \frac{t^{\text {ind } \pi \sigma}}{\prod_{1 \leqq j<n}\left(1-t^{j}\right)}=\left\{\begin{array}{l}
1 \text { for } \pi \in \mathcal{X} \\
0 \text { for } \pi \in S_{n} \backslash \mathcal{X}
\end{array}\right.
$$

Now we fix $n$ and suppose that $R$ contains a primitive $n$-th root of unity $\varepsilon$. Then $\prod_{1 \leqq j<n}\left(1-\varepsilon^{j}\right)=n$, and therefore 3.4 implies $(t=\varepsilon)$ that

$$
\sum_{\sigma \in S_{n}} e^{\mathrm{ind} \sigma} \sigma \omega_{n}=n \omega_{n}
$$

If $n$ is a unit in $R$ (which will be assumed in the sequel), then

$$
\lambda_{n}=\frac{1}{n} \sum_{\sigma \in S_{n}} \varepsilon^{\mathrm{ind} \sigma} \sigma, \quad \zeta_{n}=\frac{1}{n} \sum_{i=0}^{n-1} \varepsilon^{-i}(1, \ldots, n)^{i}
$$

are the elements of $R S_{n}$ which are called $\ell_{n}, c_{n}$ in [5]. The reader is asked to forgive our changes in notation, made for the sake of uniformity in our terminology. Moreover, it should be noted that, in [5], the product of permutations has to be read from right to left. Thus we have, in our context, by [5, Lemma 2], the following identities

$$
\lambda_{n} \zeta_{n}=\lambda_{n}, \quad \zeta_{n} \lambda_{n}=\zeta_{n}, \quad \zeta_{n}^{2}=\zeta_{n}, \quad \lambda_{n}^{2}=\lambda_{n},
$$

which imply, as in [5, Lemma 3], that 

$\zeta_{n} R S_{n}$ and $\lambda_{n} R S_{n}$ are isomorphic $R S_{n}$-right modules.

By (11), we have

$$
\lambda_{n} \omega_{n}=\omega_{n}
$$

hence

$$
\lambda_{n} u=u \text { for all } u \in \mathcal{C}(\text { see [5, Proposition 3]). }
$$

The statement (12) enables us to prove

THEOREM 3.5. $\omega_{n} \lambda_{n}=n \lambda_{n}$.

Proof: Using the preparations above and $[2,(10),(7),(9)]$, we have

$$
\mathcal{L}=\lambda_{n} \mathcal{L} \subseteq \lambda_{n} \mathcal{A}=\lambda_{n}\left(x_{1} \ldots x_{n}\right) R S_{n}=\left(x_{1} \ldots x_{n}\right) \lambda_{n} R S_{n} \cong \lambda_{n} R S_{n} \cong \zeta_{n} R S_{n} .
$$

In particular, $\lambda_{n} \mathcal{A}$ is a free $R$-module of rank $(n-1)$ !. As is easily seen (see [1, 4.8.1]), $\mathcal{L}$ is also free over $R$ of rank $(n-1)$ !. In the special case of $R=\mathbb{C}$, this implies

$$
\lambda_{n} \mathcal{A}=\mathcal{L},
$$

hence by the Specht-Wever theorem, $\omega_{n} \lambda_{n} x_{1} \ldots x_{n}=n \lambda_{n} x_{1} \ldots x_{n}$, and therefore ([2, (8)]) $\omega_{n} \lambda_{n}=n \lambda_{n}$. As this equation holds in the group ring of $S_{n}$ over $\mathbb{Z}[1 / n, \varepsilon]$, it holds in general (that is, if $R$ contains a primitive $n$-th root of unity $\varepsilon$ and $n$ is invertible in $R$ ).

The proof shows that $\mathcal{L} \subseteq \lambda_{n} \mathcal{A} \cong \zeta_{n} R S_{n}$ for every ring $R$ containing $\varepsilon$ and $n^{-1}$. From 3.5 and the Specht-Wever theorem follows that $\mathcal{L} \supseteq \lambda_{n} \mathcal{A}$ and therefore $\mathcal{L} \cong \zeta_{n} R S_{n}$, that is, (Ind).

\section{REFERENCES}

[1] Y.A. Bahturin, Identical Relations in Lie Algebras (VNU Science Press, Utrecht, 1987).

[2] D. Blessenohl and H. Laue, 'Generalised Jacobi identities', Note Mat. (to appear).

[3] M. Hall, Combinatorial theory (Wiley-Interscience, New York, 1986).

[4] G.D. James and A. Kerber, The representation theory of the symmetric group (Addison-Wesley, Reading, Mass., 1981).

[5] A.A. Klyachko, 'Lie elements in the tensor algebra', Siberian Math. J. 15 (1974), 914-920.

[6] F. Wever, 'Über Invarianten in Lie'schen Ringen', Math. Ann. 120 (1949), 563-580.

H. Laue

Dipartimento di Matematica

Università degli Studi

Via Arnesano

1-73100 Lecce

Italy.
D. Blessenohl

Mathenatisches Seminar der Universität

Ludewig-Meyn-Str. 4

D 2300 Kiel 1

West Germany 